\title{
Evaluation of microkinesitherapy effectiveness in post-traumatic cervicalgia: a new approach applied to previous data
}

\author{
Pierre Baconnier', Bruno Vial², Gérard Vaudaux², Geneviève Vaudaux², Jean-Luc Bosson ${ }^{3}$
}

\begin{abstract}
Background. Cervicalgia from traumatic events such as road traffic accidents and falls can lead to musculoskeletal and soft tissue injuries with the development of pain and headache. Microkinesitherapy is a manual therapy technique that normalizes soft tissue tensions and articular range of motion throughout micropalpation. Objective. This study is a secondary analysis with a clinical significance approach to data collected in a previously published study. These data were obtained during a randomized double-blind clinical trial to evaluate the effectiveness of microkinesitherapy treatment in cervical trauma. Methods. The authors performed a secondary analysis of a previous randomized, double-blind clinical trial, using the Minimal Clinically Important Difference (MCID). This study was conducted at the Faculty of medicine of the Université Grenoble Alpes (Grenoble, FRA), and analyzed 29 patients with cervical trauma less than three months of trauma. This is a secondary analysis of data collected in a previously published randomized double-blind clinical trial where the treated group received a microkinesitherapy treatment ( $n=15,8$ females) and the other group received a sham treatment ( $n=14,7$ females). To look at the clinical significance of those results, it was used the MCID of the instantaneous pain and cervical articular mobility, before and after the treatment or sham treatment. The clinical significance of the treatment was obtained through an inter-group comparison (Pearson's chi-squared test) based on MCID. Results. With both threshold values, the Pearson's chi-squared test provide a significant difference in the treatment result compared to sham group. Conclusion. The use of the MCID concept and its value is drawn from recent studies on neck pain allowed us to demonstrate, on an intergroup analysis basis, that there is a clinically significant difference in the effect of a microkinesitherapy treatment on post-traumatic neck pain as compared to a sham treatment.
\end{abstract}

Keywords: Neck Pain; Manual Therapy; Minimal Clinically Important Difference; Visual Analog Scale; Inter-group; Clinical Research.

\section{INTRODUCTION}

The main causes of acute post-traumatic neck pain are road traffic accidents, sports injuries and falls ${ }^{(1)}$. To our knowledge, only one double-blind assessment has been made on the effectiveness of microkinesitherapy as regards cervicalgia which evaluated the effects of a microkinesitherapy session on pain and the range of flexion-extension motion in post-traumatic acute neck pain ${ }^{(2)}$. A letter to the editor was published in response to this article ${ }^{(3)}$. This manuscript may be considered as a response to this letter. Data were obtained from a randomized double-blind clinical trial involving two groups of patients: a microkinesitherapy group that benefitted from the treatment sequence, and a sham group that had a simulation sequence. The primary outcome measure was the evolution of pain, and the secondary endpoint evaluated range of motion (ROM). The evaluations of measures were carried out before (initial check-up) and after (second check-up) treatment. The choice of the assessment of pain as the main criterion was motivated by the will to test the effectiveness of microkinesitherapy on this parameter. To detect a possible modification of the values of the two variables studied between the two check-ups for each group, the Student " $\mathrm{t}$ " test (comparison of averages of matched data) was chosen. A significant decrease in the visual analog scale (VAS) for pain as well as improvement in ROM was observed in the microkinesitherapy group, but not in the sham group for both parameters. Thus, although the data analysis technique used in the previous report based on an intragroup approach demonstrated the effectiveness of microkinesitherapy, this result did not prove that the effect of a simulation sequence was different (intergroup analysis could not demonstrate between group differences).

In the field of physiotherapy, the routine use of patient-reported outcome measures may provide an effective way of monitoring patient valued outcomes ${ }^{(4,5)}$. Indeed, statistical significance is not equivalent to clinical significance ${ }^{(6)}$. It is now well established ${ }^{(4,7,8)}$ that evaluating whether the change from baseline is important at the individual patient level and then reporting the percentage of patients with

Corresponding author: B.Vial --24, Boulevard de la Chantourne -38700 La Tronche, France- Phone: 0033670550690 - Email: vial.bruno@orange.fr

2. Boulevard de la Chantourne -38700 La Tronche, France.

Full list of author information is available at the end of the article.

Funding: This research did not receive any specific grant from funding agencies in the public, commercial, or not-for-profit sectors.

Submission date 18 September 2019; Acceptance date 29 October 2019; Publication date 21 December 2019 
the improved condition in different treatment groups is an interesting approach. This approach requires that the continuous outcome measure is dichotomized into a binary variable, with change greater or less than a cut-off defining an important improvement in the patient's symptom. Thus it is necessary to determine the cut-off value which is usually named Minimal Clinically Important Difference (MCID) ${ }^{(7)}$. Among the numerous papers on the subject, some concern the methodology needed to determine this cut-off $(6,9,11)$, and some give a value for specific clinical situations ${ }^{(6,12,14)}$. In both instances, an important point is an outcome for which the MCID is estimated. Concerning studies dealing with neck pain $^{(5,12,13,15,18)}$, a recent paper ${ }^{(18)}$ points out the weak sensitivity of MCID as regards functional scores while pain scores give better results. The present analysis has thus focused only on MCID for pain, which was the primary endpoint of the previous report. So the aim of the present report is to reassess the efficacy of a microkinesitherapy treatment in post-traumatic cervicalgia with the help of an approach that takes into account the clinical significance of the observed results rather than their statistical significance.

\section{METHODS}

This study is a secondary analysis of data collected in a previously published randomized double-blind clinical trial(2) aimed at evaluating the effectiveness of microkinesitherapy treatment in first-line cervical trauma less than three months after trauma. The procedure for double-blind was the following: the physiotherapist in charge of the treatment (microkinesitherapy or sham) could not exchange any word with the patient apart those required for his installation. The investigator, as well as the patient, did not know the treatment. The microkinesitherapy treatment details were described in the original paper ${ }^{(2)}$. The experimentation was conducted according to a research protocol with direct benefit. The advisory committee on biomedical research of Grenoble University 2 accepted this protocol on July 10, 1998 (Ref: 98 / ACDM / 1 / C2). Explorations were conducted in accordance with good clinical practice. The team involved in the study consisted of eighteen recruiting physicians, the coordinator, two investigating physicians and five physiotherapists trained in microkinesitherapy (ten years of practice minimum). The study involved 29 patients divided into a microkinesitherapy group (treated, $n=15,8$ females) who benefitted from the treatment and the sham group (sham, $\mathrm{n}=14,7$ females) that underwent a treatment simulation. The inclusion procedure and criteria are described in detail in the initial article ${ }^{(2)}$. The inclusion and exclusion criteria aimed at selecting patients with less than 3 months post-traumatic neck pain, with no previous treatment, presenting no form of other neck pain or cervicobrachial neuralgia and never treated with microkinesitherapy.
Assessment of treatment effectiveness was performed according to the evolution of two parameters: namely pain (primary outcome measure) and joint mobility (secondary endpoint), before and after treatment or simulation. The patients assessed their instantaneous pain at rest using a visual analog scale (VAS) ranging from 0 to 10 according to the protocol based on the use of a continuous linear scale ${ }^{(19)}$. For joint mobility assessment, the patients actively made flexion-extension movements, and ROM was assessed using the three axes-goniometric headset Cervicontrol ${ }^{\circledR}$ (Promokiné, Amiens, France). At inclusion, no statistical difference was observed between demographic, clinic or outcome characteristics between the two groups. After treatment (or simulation), a significant change in the VAS $(5.2 \pm 2.3$ at initial check-up versus $2.5 \pm 1.7$ in the second check-up, $\mathrm{p}<0.001)$ as well as ROM $\left(95^{\circ} \pm 29^{\circ}{ }^{\circ}\right.$ in the first check-up versus $107^{\circ} \pm 27^{\circ}$ in the second check-up, $\mathrm{p}<0.02$ ) were noted for the microkinesitherapy group, but no significant change in the sham group for both outcomes (VAS: $4.0 \pm 2.3$ at initial check-up versus $3.1 \pm 2.4$ in the second check-up, $p=0.23$ and ROM: $104^{\circ} \pm 26^{\circ}{ }^{\circ}$ in the first check-up versus $107^{\circ} \pm 28^{\circ}$ in the second check-up, $\mathrm{p}=0.26$ ) .

The MCID approach based on a threshold value validated for neck pain as evaluated by patients was used in order to look at the clinical significance of those results. This analysis is a post hoc one, as it was not planned in the initial study. The choice of the cut-off value has been carried out by looking at studies dealing with neck pain ${ }^{(12,13,16,20)}$ and/or physical therapy for treat neck pain ${ }^{(5,12)}$. The closest study to the present one, a systematic review of randomized control trials on whiplash conservative management ${ }^{(20)}$, revealed an $\mathrm{MCll}$ value for pain intensity of $20 \mathrm{~mm}$ (on a $0-100 \mathrm{~mm}$ scale). This value is in the range of those given by other authors, from 1.3 to 3.5 (on a 0-10 scale). Another cut-off value (1.5) has been tested as defined by a reference paper reporting results of a multinational study involving 1,532 patients suffering from various musculoskeletal diseases $^{(9)}$. In each group (treated and sham) we counted the number of VAS change (pre-post treatment) larger than the 2.0 cut-off value and tested the difference in the effect of treatment with the Pearson chi-squared test. This was also done with the 1.5 cut-off value.

\section{RESULTS}

Table 1 presents the contingency table: noting the number of improved (VAS change $>2.0$ ) and unimproved patients by treatment group as obtained from previous study data. The Pearson's chi-squared test value, risk difference, risk ratio and number needed to treat (NNT) are also given. In both cases, the Pearson's chi-squared test yielded a significant difference $(p<0.05)$ in the treatment result between the two groups. Table 2 presents the same data with a cut-off value at 1.5. The conditions of validity of the Pearson chi-squared test (all numbers in boxes of the expected table over 5 ) are satisfied for both tests. 
Table 1. Contingency table and Pearson's Chi-squared test for MCII=2.0

\begin{tabular}{lcc}
\hline & \multicolumn{2}{c}{ Group } \\
\cline { 2 - 3 } & treated & Sham \\
\hline Improved & $10(66.7 \%)$ & $3(21.4 \%)$ \\
Not improved & $5(33.3 \%)$ & $11(78.6 \%)$ \\
Total & $15(100 \%)$ & $14(100 \%)$ \\
\hline
\end{tabular}

Note: Pearson's Chi-squared $=5.9919, \operatorname{Pr}=0.014$; Risk difference $(R D)=(10 / 15)$ $(3 / 14)=0.45$ [0.13-0.77]; Risk Ratio $(R R)=(10 / 15) /(3 / 14)=3.11$ [1.07-9.02] ; Number Needed to Treat $(N N T)=1 / R D=2.21$ i.e. 3 subjects.

Table 2. Contingency table Pearson's Chi-squared test for MCII=1.5

\begin{tabular}{lrc}
\hline & \multicolumn{2}{c}{ Group } \\
\cline { 2 - 3 } & Treated & Sham \\
\hline Improved & $11(73.3 \%)$ & $535.7 \%)$ \\
Not improved & $4(26.7 \%)$ & $9(64.3 \%)$ \\
Total & $15(100 \%)$ & $14(100 \%)$ \\
\hline
\end{tabular}

Note: Pearson's Chi-squared $=4.1435, \operatorname{Pr}=0.042 ;$ Risk Difference $(R D)=(11 / 15)$ $(5 / 14)=0.38$ [0.04-0.71]; Risk Ratio $(R R)=(11 / 15) /(5 / 14)=2.05$ [0.95-4.42]; Number Needed to Treat $(N N T)=1 / R D=2.66$ i.e. 3 subjects

\section{DISCUSSION}

Neck pain is one of the leading causes of disability in the world $^{(1)}$, and trauma is an important precipitating factor. Complementary and alternative treatments of neck pain (microkinesitherapy excepted), have generally been found to be superior to no treatment, but the evidence that they are superior to sham treatments or other treatments is weak, negative, or conflicting ${ }^{(1)}$. The therapeutic effects of microkinesitherapy are not much investigated. A recent experimental study demonstrated the possible efficacy of microkinesitherapy in treating the underlying mechanisms of acute stress $^{(21)}$. In the context of the present study, the aim of microkinesitherapy is, to cure post-traumatic pain by easing muscle tension induced by trauma and thus to be a potential adjuvant for post-traumatic neck pain treatment. Few clinical trials have evaluated treatments for neck pain ${ }^{(1)}$ and our previous paper is the only double-blind assessment made on the effectiveness of microkinesitherapy in this specific context. The present study is based on these data obtained through a monocentric double-blind prospective study in two groups (treated and sham). The small size of the population finishing the study may be explained by the difficulties of recruitment in the context of private practice. The reduced number of participants could have resulted in limiting the statistical strength of the tests. In the previous report, an intragroup approach demonstrated the statistically significant effect of microkinesitherapy, but the statistical intergroup analysis could not prove that the effect of simulation sequence was different. In the present, reports a post hoc approach, based on a comparison of the clinical significance of treatment results versus sham results, demonstrated a significant difference between microkinesitherapy and sham treatment (intergroup analysis) effects on pain as evaluated by each patient. This result is obtained through the use of the Minimal Clinically Important Difference (MCID), an established concept recently applied to physiotherapy ${ }^{(4,5)}$. The values of $\mathrm{MCll}$ for pain considered in the present study ( 2.0 and 1.5 on a $0-10$ scale) are commonly used in neck pain studies $(\mathrm{MCID}=2.0)^{(20)}$ as well a more generic clinical situation $(M C I D=1.5)^{(10)}$. The lower value for MCID (1.3) given in other studies ${ }^{(5,16)}$ has been rejected as it is lower than the minimum detectable change $(M D C=2.0)$ in these studies. The intergroup analysis used based on the Pearson's Chi-squared test demonstrates a significant difference while the average change in pain VAS interpreted with MCID was not significant: averaged VAS change was $2.8(>2.0)$ in the treated group and $0.9(<1.5)$ in the sham group. Taking into account confidence intervals (respectively \pm 2.1 and \pm 2.6 ), these average values were not statistically higher or smaller than any of the 2 MCID's, namely 1.5 or 2.0 .

\section{CONCLUSION}

In conclusion, the use of the MCID concept and its value is drawn from recent studies on neck pain allowed us to demonstrate, on an intergroup analysis basis, that there is a clinically significant difference in the effect of a microkinesitherapy treatment on post-traumatic neck pain as compared to a sham treatment. The relevance of this study must be put into perspective because of the reduced number of patients included. Therefore, we believe that a trial carried out under the same conditions on a larger population cohort and over a period of at least three months could provide more convincing results. This needs to diversify the specialties of recruiting physicians (rheumatologists, sports doctors, general practitioners, hospital and clinical emergency services) and extend the recruitment in a multicenter approach.

\section{AUTHORS CONTRIBUTIONS}

PB: statistical analysis and scientific advice for manuscript writing; BV: concept of experimental design, physiotherapist; Gérard V coordinator, concept of experimental design, physiotherapist; Geneviève $V$ concept of experimental design, physiotherapist; JLB: statistical analysis and scientific advice for manuscript writing.

\section{CONFLICTS OF INTEREST}

The authors declare no conflict of competing interest regarding this study.

\section{AUTHORS DETAILS}

1. Université Grenoble Alpes - UMR 5525 - TIMC PRETA Lab - CNRS UGA Faculty of Medicine- F-38041 Grenoble, France. 3. Université Grenoble Alpes - UMR 5525 - ThEMASLab - CNRS UGA - Faculty of Medicine - F-38041 Grenoble, France. 


\section{REFERENCES}

1. Cohen PS. Epidemiology, Diagnosis, and Treatment of Neck Pain. Mayo Clin Proc. 2015; 90: 284-299.

2. Baconnier P, Vial B, Vaudaux G, Vaudaux, G, Maindet-Dominici C; Poquin $D$ et al. Evaluation of the clinical effectiveness of microkinesitherapy in post-traumatic cervicalgia. A randomized, double-blinded clinical trial. MTP \& Rehab Journal. 2016; 14: 385-9.

3. Pinsault N, Dolgopoloff M, Guillaud A. Letter to the Editor.MTP \& Rehab Journal; 2017; 15: 497

4. Kyte DG, Clavert M, Van der Wees PJ, ten Hover R, Tolan S, Hill JC. An introduction to patient-reported outcome measures (PROMs) in physiotherapy. Physiotherapy. 2015; 101: 119-25.

5. Horn ME, Brennan GP, George SZ, Harman JS, Bishop MD. A value proposition for early physical therapist management of neck pain: a retrospective cohort analysis. BMC Health Services Research. 2016; 16 : 253-61.

6. Wu X, Liu J, Tanadini LG, Wu X, Liu J, Lammertse DP et al. Challenges for defining minimal clinically important difference (MCID) after spinal cord injury. Spinal Cord. 2015; 53(2): 84-91.

7. Jeschke R, Singer J, Guyatt GH. Measurement of health statusascertaining the minimal clinically important difference. Control Clin Trials.1989;10:407-15.

8. Tubach F, Ravaud P. Minimal clinically important difference and patient acceptable symptomatic state, two concepts to help interpretation of clinical trial results. La lettre du Rhumatologue. 2006; 321: 3-4.

9. Crosby RD, Kolotkin RL, Williams R. Defining clinically meaningful change in health-related quality of life. J Clin Epidemiol. 2003; 56:395-407.

10. Tubach F, Ravaud P, Martin-Mola E, Awada H, Bellamy N, Bombardier $C$, et al. Minimum clinically important improvement and patient acceptable symptom state in pain and function in Rheumatoid Arthritis, ankylosing spondylitis, Chronic Back Pain, Hand Osteoarthritis, and Hip and Knee Osteoarthritis: Results from a Prospective Multinational Study. Arthr Care \& Res. 2012; 11(64): 1699-707.
11. Katz P, Paillard C, Ekman E. Determining the clinical importance of treatment benefits for interventions for painful orthopedic conditions. $J$ Orthop Surg Res. 2015; 10: 24-34.

12. Carreon LY, Glassman SD, Campbell MJ, Anderson PA. Neck Disability Index, short form-36 physical component summary, and pain scales for neck and arm pain: the minimum clinically important difference and substantial clinical benefit after cervical spine fusion. Spine. 2010; 10(6): 469-74.

13. Juul T, Søgaard K, Davis AM, Roos EM. Psychometric properties of the Neck Outcome Score, Neck Disability Index and Short Form-36 were evaluated in patients with neck pain. J Clin Epidemiol. 2016; 79: 31-40.

14. Myles PS, Myles DB, Galagher W, Boyd D, Mastigação C, MacDonald $\mathrm{N}$, et al. Measuring acute postoperative pain using the visual analog scale: the minimal clinically important difference and patient acceptable symptom state. Br J Anaesth. 2017;118(3): 424-9.

15. Pool JJM, Ostelo RWJG, Hoving JL, Bouter LM, Vet HCW. Minimal clinically important change of the Neck Disability Index and the Numerical Rating Scale for patients with neck pain. Spine. 2007; 32(26): 3047-51.

16. Cleland JA, Childs JD, Whitman JM. Psychometric properties of the Neck Disability Index and numeric pain rating scale in patients with mechanical neck pain. Arch Phys Med Rehabil. 2008 ;89:69-74.

17. Jørgensen R, Ris I, Falla D, Juul-Kristensen B. Reliability, construct and discriminative validity of clinical testing in subjects with and without chronic neck pain. BMC Musculoskeletal Disorders. 2014; 15: 408-22.

18. Jørgensen R, Ris I, Juhl C, Falla D, Juul-Kristensen B. Responsiveness of clinical tests for people with neck pain. BMC Musculoskeletal Disorders. 2017; 18: 548-55.

19. Bodian CA, Freedman G, Hossain S, Eisenkra JB, Beilin Y. The visual analog scale for pain: clinical significance in postoperative patients. Anesthesiology. 2001; 95(6): 1356-61.

20. Wiangkham T, Duda J, Haque S, Madi M, Rushton A. The effectiveness of conservative management for acute whiplash-associated disorder (WAD) II: a systematic review and meta-analysis of randomized controlled trials. PLoS One. 2015; 10(7): e0133415.

21. Grosjean D, Salgado A, Parreira R, et al. A new approach to manual therapy for the immune system: an experimental study. Int J Sci Res Methodol. 2017; 8(1): 137-48. 